\title{
True 99th centile of high sensitivity cardiac troponin for hospital patients: prospective, observational cohort study
}

\author{
Mark Mariathas, ${ }^{1}$ Rick Allan, ${ }^{2}$ Sanjay Ramamoorthy, ${ }^{3}$ Bartosz Olechowski, ${ }^{1}$ Jonathan Hinton, ${ }^{1}$ \\ Martin Azor, ${ }^{4}$ Zoe Nicholas, ${ }^{1}$ Alison Calver, ${ }^{5}$ Simon Corbett, ${ }^{5}$ Michael Mahmoudi, ${ }^{1,5}$ \\ John Rawlins, ${ }^{5}$ Iain Simpson, ${ }^{5}$ James Wilkinson, ${ }^{5}$ Chun Shing Kwok, ${ }^{6}$ Paul Cook, ${ }^{2}$ \\ Mamas A Mamas, ${ }^{6}$ Nick Curzen ${ }^{1,5,7}$
}

For numbered affiliations see end of the article.

Correspondence to: N Curzen, E Level North Wing, University Hospital Southampton NHS Trust, Southampton SO16 6YD, UK nick.curzen@uhs.nhs.uk (or @NickCurzen on Twitter ORCID 0000-0001-9651-7829)

Additional material is published online only. To view please visit the journal online.

Cite this as: $B M / 2019 ; 364: 1729$ http://dx.doi.org/10.1136/bmj.l729

Accepted: 08 February 2019

\section{ABSTRACT}

OBJECTIVE

To determine the distribution, and specifically the true 99th centile, of high sensitivity cardiac troponin I (hs-cTnl) for a whole hospital population by applying the hs-cTnl assay currently used routinely at a large teaching hospital.

\section{DESIGN}

Prospective, observational cohort study.

SETTING

University Hospital Southampton NHS Foundation Trust, Southampton, United Kingdom, between 29 June 2017 and 24 August 2017.

\section{PARTICIPANTS}

20000 consecutive inpatients and outpatients undergoing blood tests for any clinical reason. Hs-cTnl concentrations were measured in all study participants and nested for analysis except when the supervising doctor had requested hs-cTnl for clinical reasons.

\section{MAIN OUTCOME MEASURES}

Distribution of hs-cTnl concentrations of all study participants and specifically the 99th centile.

RESULTS

The 99th centile of hs-cTnl for the whole population was $296 \mathrm{ng} / \mathrm{L}$ compared with the manufacturer's quoted level of $40 \mathrm{ng} / \mathrm{L}$ (currently used clinically as the upper limit of normal; ULN). Hs-cTnI

\section{WHAT IS ALREADY KNOWN ON THIS TOPIC}

Current guidelines recommend the use of troponin assays to help exclude or diagnose acute myocardial infarction

Manufacturers of troponin assays provide a recommended 99th centile that is based on a few hundred healthy individuals; this level is often used as the upper limit of normal when applied to the hospital population

A variety of clinical factors affect the troponin level, such as age, sex, and renal function, but little is known about the true distribution of the troponin level across the whole hospital population

\section{WHAT THIS STUDY ADDS}

In a hospital population of 20000 consecutive patients, one in 20 of all patients had a high sensitivity troponin I concentration greater than the manufacturer's recommended 99th centile; in most of these patients there was no clinical suspicion of acute myocardial infarction

It is important to interpret the troponin result in hospital patients according to individual patients, their clinical presentation, and the guideline recommendations for correct diagnosis of type 1 and type 2 myocardial infarction These results could help to avoid misdiagnosis and inappropriate treatment concentrations were greater than $40 \mathrm{ng} / \mathrm{L}$ in one in $20(5.4 \%, n=1080)$ of the total population. After excluding participants diagnosed as having acute myocardial infarction $(n=122)$ and those in whom hs-cTnl was requested for clinical reasons $(n=1707)$, the 99 th centile was $189 \mathrm{ng} / \mathrm{L}$ for the remainder $(n=18171)$. The 99th centile was $563 \mathrm{ng} / \mathrm{L}$ for inpatients $(\mathrm{n}=4759)$ and $65 \mathrm{ng} / \mathrm{L}$ for outpatients $(n=9280)$. Patients from the emergency department $(n=3706)$ had a 99 th centile of $215 \mathrm{ng} / \mathrm{L}$, with $6.07 \%$ $(n=225)$ greater than the recommended ULN. 39.02\% $(n=48)$ of all patients from the critical care units $(n=123)$ and $14.16 \%(n=67)$ of all medical inpatients had an hs-cTnl concentration greater than the recommended ULN.

\section{CONCLUSIONS}

Of 20000 consecutive patients undergoing a blood test for any clinical reason at our hospital, one in 20 had an hs-cTnl greater than the recommended ULN. These data highlight the need for clinical staff to interpret hs-cTnl concentrations carefully, particularly when applying the recommended ULN to diagnose acute myocardial infarction, in order to avoid misdiagnosis in the absence of an appropriate clinical presentation.

\section{TRIAL REGISTRATION}

Clinicaltrials.gov NCT03047785.

\section{Introduction}

The use of increasingly sensitive troponin assays for excluding or diagnosing acute myocardial infarction has become universal. A diagnosis of acute myocardial infarction is defined, in the context of an appropriate clinical presentation, by a rise or fall in cardiac troponin concentration, now the gold standard biomarker, ${ }^{12}$ with at least one value greater than the 99th centile derived from a reference population of healthy individuals..$^{3-5}$

Under most circumstances, the troponin assay is requested by frontlineclinicalstaff todetermine whether a patient is having a type 1 myocardial infarction caused by coronary plaque rupture or erosion. Robust evidence has shown symptomatic and prognostic benefit from applying early pharmacological and interventional treatment strategies in these patients. However, particularly with the advent of newer assays, this strategy has two potential challenges.

Firstly, raised cardiac troponin concentrations, particularly in patients not presenting with a typical history of cardiac pain, are often caused by myocardial injury or type 2 myocardial infarction. ${ }^{6}$ These 
conditions, which are secondary to ischaemia caused by increased oxygen demand or decreased supply rather than a plaque erosion, ${ }^{8-10}$ are not well recognised when the troponin test is requested or the result interpreted. Correct diagnosis is important because most patients with type 2 myocardial infarction have not been shown to benefit from the same aggressive pharmacotherapy and invasive investigation and treatment that are offered as standard in patients with type 1 myocardial infarction. ${ }^{11}$ Some exceptions include spontaneous coronary dissection, coronary embolism, and coronary spasm. ${ }^{10} 12$ Misinterpretation may lead to inappropriate management, including prolonged antiplatelet therapy and invasive coronary angiography, with or without revascularisation.

Secondly, the assay specific 99th centile (upper limit of normal; ULN) is generally applied as a binary "rule in" or "rule out" threshold for acute myocardial infarction. Recent trial data confirm the veracity of using early cardiac troponin concentrations to confidently exclude a diagnosis of acute myocardial infarction ${ }^{13-16}$; however, the assumption that a concentration greater than the recommended threshold implies acute myocardial infarction (and in particular type 1 myocardial infarction) is often inappropriate.

Both of these potential issues may be compounded in clinical practice by the increasing sensitivity of the available assays that are able to detect troponin at much lower concentrations than previously. ${ }^{5}$ Consequently, new highly sensitive cardiac troponin (hs-cTn) assays ${ }^{17-21}$ allow for rapid exclusion of acute myocardial infarction, and thereby enable patients to be discharged early from hospital. Furthermore, modern hs-cTn assays can detect troponin in more than $50 \%$ of the general population, with some assays able to detect troponin in everyone. ${ }^{22}$ The appropriate interpretation of raised hs-cTn, specifically in relation to the diagnosis of type 1 myocardial infarction, is therefore dependent on a clinical presentation consistent with this diagnosis, and, in particular, a history of cardiac-sounding chest pain, according to the guidelines.

The International Federation of Clinical Chemistry and Laboratory Medicine Task Force on Clinical Applications of Bio-Markers currently recommends that the 99th centile for any assay can be calculated using 300 "healthy" men and 300 "healthy" women. ${ }^{23}$ However, several factors are known to affect an individual's troponin, ${ }^{23}$ including age, ${ }^{24}$ sex, $^{25}$ glomerular filtration rate, ${ }^{26}$ left ventricular function, ${ }^{27}$ and the presence of major inflammatory conditions. ${ }^{28}$ Therefore, whether the clinically applied concept of a ULN for the hs-cTn assay is appropriate requires closer scrutiny, particularly when it has been derived from a limited number of healthy individuals. Importantly, the approaches to determining the recommended 99th centile are also variable. ${ }^{29-31}$

The objective of the CHARIOT study was to determine the true distribution of the highly sensitive cardiac troponin I (hs-cTnI) concentration, and more specifically the 99th centile, in a population of consecutive inpatients and outpatients in our hospital. Our hypothesis was that the true 99th centile of hs-cTnI in this population would differ from the manufacturer recommended ULN for this assay. This difference would highlight the potential for misinterpretation of a concentration greater than this threshold in routine clinical practice, particularly when making a diagnosis of acute myocardial infarction and especially type 1 myocardial infarction.

\section{Methods}

\section{Study population}

This was a prospective, observational study of 20000 consecutive patients aged at least 18 years in whom a biochemistry blood test was requested for clinical reasons by their supervising doctor at our institution, University Hospital Southampton (United Kingdom). It was conducted between 29 June 2017 and 24 August 2017. We included patients regardless of the setting in which the blood test was requested. Therefore, the study population consisted of outpatients and inpatients, attendees at the emergency department, elective and emergency admissions, and every specialty within the hospital. For each patient included in the study, only one troponin measurement was performed on the first biochemistry blood sample that became available during the study period. That patient was then excluded from further sampling so that a consecutive series of 20000 different patients were included. During some of our analyses, we excluded patients in whom a troponin was requested for clinical reasons by the supervising doctor, and those in whom a diagnosis of acute myocardial infarction was made. This was determined by reviewing the electronic blood request forms submitted to the biochemistry department and by electronic discharge summaries.

\section{Approvals}

As part of the ethical committee process, we sought approval from the Confidentiality Advisory Group based on two unusual aspects of the methods. Firstly, patients did not know that an extra blood assay was being performed and consent was not sought or required. Secondly, except for patients who had an hs-cTnI test as part of their routine clinical care as requested by their supervising doctor, test results were nested and not revealed to either patients or their supervising clinical team; this was regardless of whether the level was greater than the recommended ULN.

\section{Cardiac troponin I assay}

The Beckman Coulter Access AccuTnI+3 assay (Brea, CA, USA) is used in routine clinical practice at our hospital. We applied this assay to measure hs-cTnI concentrations in the study population. The manufacturer's recommended 99th centile (ULN) is $40 \mathrm{ng} / \mathrm{L}$, which is the level we use in routine clinical practice. The coefficient of variation of the assay is less than $10 \%$ at $40 \mathrm{ng} / \mathrm{L}$; the limit of quantification $(10 \%$ of the coefficient of variation) is $20 \mathrm{ng} / \mathrm{L}$; the limit of 
detection is $8 \mathrm{ng} / \mathrm{L}$; and the limit of blank is $5 \mathrm{ng} / \mathrm{L}$. For patients in whom troponin had not been requested for clinical reasons, we measured the hs-cTnI level using serum that was surplus to clinical need. An automated, bespoke system was installed in biochemistry to ensure that each patient was only included once in the study. We collected serum in serum separator tubes and stored it at room temperature for up to 24 hours before hs-cTnI levels were measured using the DxI800 platform (Beckman Coulter). We performed quality control of the assay on a daily basis, which is routine in clinical practice.

\section{Data collection}

The baseline demographic data collected for the study were limited to those derived from electronic request forms for blood tests and, for inpatients, from electronic discharge summary codes. These data, together with the troponin levels and other study data, were saved on a bespoke database for later analysis.

\section{Statistical analysis}

We defined the 99th centile for the study population using a non-parametric procedure based on frequency tables. Statistical analyses were performed using IBM SPSS version 22.0 (Armonk, New York, NY, USA). We used Stata 14.0 (College Station, TX, USA) to perform multiple logistic regressions to identify factors associated with highly sensitive troponin levels greater than $40 \mathrm{ng} / \mathrm{L}$. Variables in the model included age, male sex, serum sodium, estimated glomerular filtration rate, and location when the biochemistry test was requested.

\section{Patient and public involvement}

The British Cardiac Patients Association assisted the researchers in reviewing the study protocol, particularly with reference to the lack of consent from participants. As part of our study application, a letter of support for our methods from the chairman of the association was sent to the Health Research Authority and the Confidentiality Advisory Group.

\section{Results}

A total of 20000 consecutive patients were included in the CHARIOT study between 29 June 2017 and 24 August 2017. The median age was 61 (standard deviation 20) and 52.9\% were women ( $\mathrm{n}=10580)$.

The 99th centile hs-cTnI concentration for the whole study population ( $\mathrm{n}=20000)$ was $296 \mathrm{ng} / \mathrm{L}$, with one in $20(5.4 \% ; n=1080)$ patients having an hscTnI concentration greater than the manufacturer's recommended ULN (40 ng/L). When we excluded all patients diagnosed as having acute myocardial infarction on discharge from hospital or in whom an hs-cTnI test had been requested for clinical reasons, 18171 patients remained. The 99th centile in these patients was $189 \mathrm{ng} / \mathrm{L}$, with $4.6 \%(\mathrm{n}=836)$ having a level greater than $40 \mathrm{ng} / \mathrm{L}$ (fig 1). Table 1 presents the baseline characteristics.

Of the 1707 patients in whom hs-cTnI concentrations were requested by the clinical team, 73\% $(n=1246)$ had presented with chest pain; arrhythmia $(n=52)$ and suspected blackouts $(n=63)$ were the next most common reasons for the test.

\section{Patient location}

We stratified patients according to their location when the biochemistry test was requested. Specifically, the study included 9280 (51.1\%) hospital outpatients in whom the observed 99th centile was $65 \mathrm{ng} / \mathrm{L}$, with hs-cTnI concentrations greater than the recommended ULN in $2 \%(n=186)$. There were $4759(26.2 \%)$ inpatients and the 99th centile for this group was 563 $\mathrm{ng} / \mathrm{L}$; the hs-cTnI concentration was greater than the recommended ULN in $7.29 \%(n=347)$.

A total of 5708 patients had their blood sampling in the emergency department. Of this group, 1551 (27.2\%) had hs-cTnI concentrations requested by doctors in the department. The 99th centile for the remaining emergency department population $(n=3706)$ was $215 \mathrm{ng} / \mathrm{L}$, with $6.07 \% \quad(n=225)$ having hs-cTnI concentrations greater than the recommended ULN. Of patients managed in the resuscitation room of the emergency department $(n=426), 19.48 \%(n=83)$ had hs-cTnI concentrations greater than the ULN.

In the critical care setting (three intensive care units and two high dependency units; $n=123$ ), 39.02\% $(n=48)$ had hs-cTnI concentrations greater than the ULN. When we excluded all patients diagnosed as having myocardial infarction or who had an hs-cTnI test requested by the clinical team, 14.16\% $(n=67)$ of all medical inpatients (excluding those on cardiac wards) had an hs-cTnI concentration greater than the recommended ULN. For the medicine for older people wards, $20.8 \%(n=20)$ had an hs-cTnI concentration greater than the recommended ULN; for patients managed on the acute surgical unit, the corresponding figures were $4.62 \% \quad(n=16)$, and for those on orthopaedic wards, $5.24 \%(n=13)$. In none of these patients was an acute myocardial infarction suspected or diagnosed (table 2; fig 2).

\section{Age}

There was an association between increasing age and distribution of troponin concentration. Supplementary tables 1 and 2, and figure 3 show centiles (25th, 50th, 75th, and 99th) and proportion of patients with hscTnI greater than the ULN according to age.

\section{Sex}

The 99th centiles for men and women were 373 and 236 $\mathrm{ng} / \mathrm{L}$, respectively. A total of $6.6 \%(\mathrm{n}=622)$ of men and $4.38 \%(n=463)$ of women had hs-cTnI concentrations greater than the ULN. Significant differences were seen in mean hs-cTnI levels when comparing men with women (62 v $31 \mathrm{ng} / \mathrm{L}, \mathrm{P}=0.021)$.

\section{Multivariable analysis}

When we excluded all patients who had been diagnosed with myocardial infarction or had hscTnI tests requested by the clinical team $(n=1829)$, a 

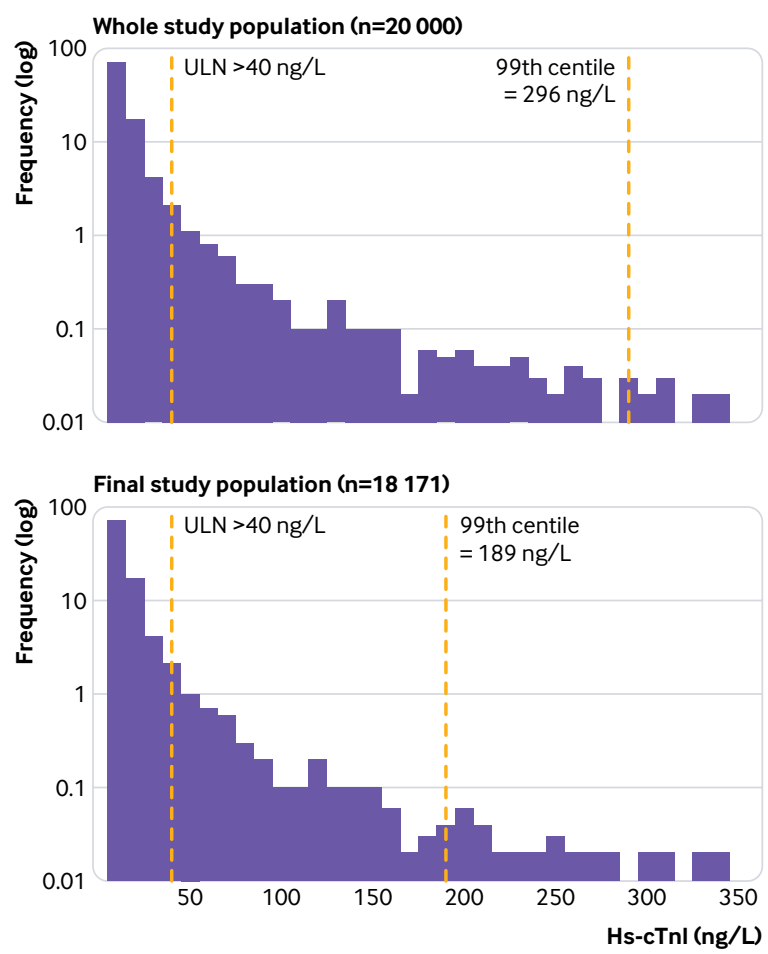

Fig 1 | Log distribution of high sensitivity cardiac troponin I (hs-cTnl) concentration in whole study population $(n=20000)$ and in final study population $(n=18171)$. ULN=manufacturer's recommended upper limit of normal for hs-cTnl concentration $(>40 \mathrm{ng} / \mathrm{L})$

\section{Discussion}

In this large study, we found that one in 20 consecutive inpatients and outpatients at a large UK hospital had a troponin level greater than the manufacturer recommended 99th centile (ULN) for the assay. We also showed that the true 99th centile varies according to the clinical setting, age and sex of the patient, and location when the biochemistry test was requested. Two per cent of outpatients and 39\% of patients in critical care units had a hs-cTnI concentration greater than the recommended ULN.

These results have important clinical implications that are almost certainly relevant to the application of all modern hs-cTn assays. Firstly, they confirmed our hypothesis that the true 99th centile for a general hospital population is not consistent with the recommended ULN. Secondly, these data raise important questions about the applicability of the quoted ULN as an arbiter of type 1 acute myocardial infarction in patients who do not give a typical history consistent with this diagnosis. Previous evidence for using "negative" hs-cTnI levels to "rule out" acute myocardial infarction is clear cut and robust. ${ }^{14-1632}$ The Fourth Universal Definition ${ }^{3}$ recommends a diagnosis of acute myocardial infarction when there is clinical evidence of acute myocardial ischaemia and when an increase or decrease in cardiac troponin levels is detected. However, using the recommended ULN as a "rule in" test for acute myocardial infarction might not be appropriate in patients presenting with atypical symptoms and other comorbidities, such as in the emergency department or on acute medical and surgical wards. This approach could expose patients to inappropriate pharmacological and invasive treatments that have only been shown to be beneficial in true type 1 myocardial infarction populations.

These data demonstrate the importance of interpreting hs-cTnI results with caution in individual patients. The risk of potential systematic misdiagnosis of acute myocardial infarction is particularly shown by the observed 99th centile for hs-cTnI in our emergency department population (215 ng/L) and acute medical admissions (1459 ng/L). In addition, about $40 \%$ of patients in some clinical settings have hs-cTnI levels greater than the recommended ULN. It is important for frontline clinical staff to understand that using a single cutoff of hs-cTnI to diagnose acute myocardial infarction might be inappropriate and that the ULN of the assay depends on the setting and the clinical characteristics of patients. We would advocate that

\begin{tabular}{|c|c|c|c|}
\hline & Hs-cTnI <ULN $(n=18915)$ & Hs-cTnl > ULN $(n=1085)$ & $P$ value \\
\hline Age (years) & 57.4 & 74.2 & $<0.001$ \\
\hline Male sex (No, \%) & $8796(46.5)$ & $622(57.3)$ & 0.005 \\
\hline $\mathrm{eGFR}\left(\mathrm{mL} / \mathrm{min} / 1.73 \mathrm{~m}^{2}\right)$ & 79.1 & 59.6 & $<0.001$ \\
\hline Sodium (mmol/L) & 137.2 & 136 & $<0.001$ \\
\hline Inpatients (No, \%) & $4540(24.0)$ & 405(37.3) & $<0.001$ \\
\hline Outpatients (No, \%) & $9155(48.4)$ & 189(17.4) & $<0.001$ \\
\hline
\end{tabular}




\begin{tabular}{|c|c|c|c|c|c|c|}
\hline Location & No of patients & Median (ng/L) & $\begin{array}{l}\text { Interquartile } \\
\text { range }(\mathrm{ng} / \mathrm{L})\end{array}$ & $\begin{array}{l}\text { Range } \\
\text { (ng/L) }\end{array}$ & $\begin{array}{l}\text { Proportion >ULN } \\
(\%(\mathrm{No}))\end{array}$ & $\begin{array}{l}\text { 99th centile } \\
\text { (ng/L) }\end{array}$ \\
\hline Outpatients & 9280 & 5 & 8 & 3255 & $2.02(187)$ & 65 \\
\hline Emergency department & 3706 & 7 & 9 & 6106 & $6.07(225)$ & 215 \\
\hline Resuscitation room & 426 & 11 & 24 & 10979 & $19.48(83)$ & 1839 \\
\hline Acute surgical unit & 346 & 6 & 9 & 2668 & $4.62(16)$ & 92 \\
\hline Medical wards & 473 & 12 & 22 & 8807 & $14.16(67)$ & 1459 \\
\hline $\begin{array}{l}\text { Medicine for older people } \\
\text { wards }\end{array}$ & 96 & 20 & 27 & 3508 & $20.83(20)$ & - \\
\hline Orthopaedic wards & 248 & 8 & 9 & 402 & $5.24(13)$ & 184 \\
\hline
\end{tabular}

clinical staff are aware of the current guidelines for diagnosing acute myocardial infarction, which are not always adhered to, and also that they have a very clear indication for requesting the test.

Our analysis highlighted several factors that are associated with raised hs-cTnI levels according to the recommended threshold, including mode of presentation. We found that $7.29 \%$ of all inpatients in this study had a raised hs-cTnI concentration, including $6.07 \%$ of the emergency department population and $19.48 \%$ of patients admitted to the resuscitation room. It is more predictable that nearly $40 \%$ of patients admitted to a critical care setting have an elevated concentration. In addition, the observed 99th centile for hs-cTnI concentrations was $65 \mathrm{ng} / \mathrm{L}$ in outpatients, and $2 \%$ of these patients who attended the hospital only for an outpatient clinic appointment had a concentration greater than the recommended ULN. These results highlight the need for a review of the distribution of the hs-cTn assay in a hospital setting. Further research is also required to determine whether there is an association between absolute troponin concentration and cardiovascular outcome in such populations.

Other factors that were clearly associated with increasing hs-cTn concentrations were age and sex. Specifically, we found that almost double the

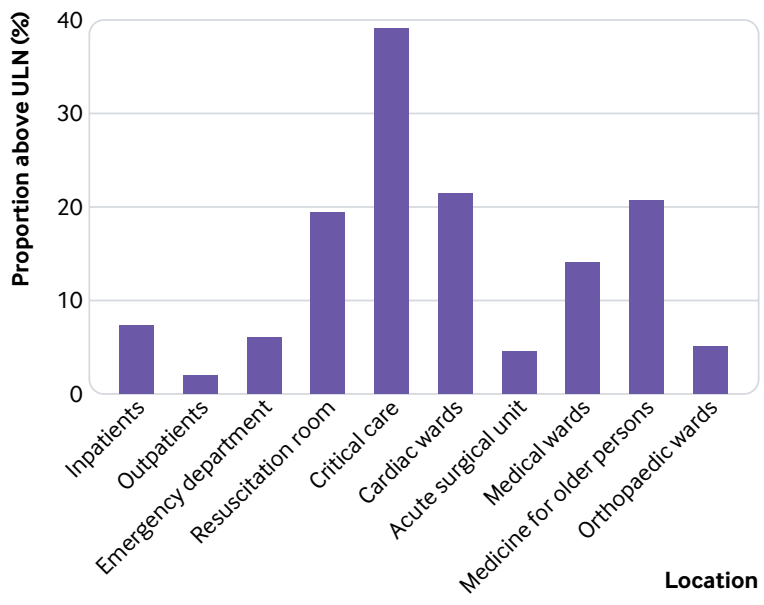

Fig 2 | Proportion of patients with high sensitivity cardiac troponin I concentration greater than manufacturer's recommended upper limit of normal (ULN=40 ng/L), according to location when the biochemistry test was requested proportion of patients in their 60s had hs-cTnI concentrations greater than the ULN compared with patients in their 50s. In addition, levels tended to be higher in men than in women. These observations lend weight to the concept that there should be age and sex specific recommendations for the ULN.

\section{Comparison with other studies}

Previous literature in this field has confirmed the use of the newer hs-cTn assays for early exclusion of acute myocardial infarction in a robust and safe manner. ${ }^{14-16} 32$ However, interpretation of a single hs-cTnI concentration above the supplied ULN as being an indicator of acute myocardial infarction, and, more specifically, type 1 myocardial infarction, by frontline clinical staff could lead to misdiagnosis and inappropriate investigations and treatment. Our data indicate that the prevalence of troponin levels above the supplied ULN in an important proportion of patients in whom there is no clinical suspicion of acute myocardial infarction should raise a cautionary note.

Our findings raise important and interesting questions about the potential implications of the observed distribution of hs-cTnI in the hospital population. Specifically, are the levels that we found in these patients, for whom the suspicion of acute myocardial infarction is low (for example, outpatients), actually abnormal? Do the levels indicate myocardial injury in their own right, and if so, are they associated with adverse outcome, perhaps as biomarkers for future cardiovascular risk? An accumulating body of evidence suggests that hs-cTn concentrations in populations of patients with stable chronic disease states, of cardiac and non-cardiac origin, are associated with risk of cardiovascular events. ${ }^{33-42}$ Notably, in the outpatient population it has been reported that hs-cTnI has been shown to be associated with an increased risk of vascular events and all cause mortality. ${ }^{4344}$ It is conceivable that the raised hs-cTn concentrations in a patient with stable disease always indicates myocardial injury or unwellness: the so-called "never means nothing" hypothesis. ${ }^{45}$

\section{Implications of this study}

The results of the CHARIOT study have important clinical implications that might be relevant to the 


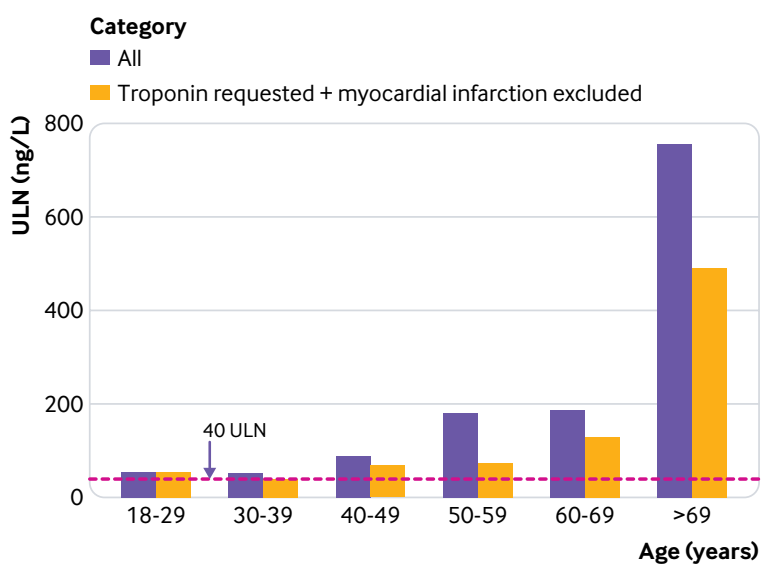

Fig 3 | Upper limit of normal concentration of high sensitivity cardiac troponin I (ULN=40 ng/L) according to age of patients

application of all modern hs-cTn assays. The notion of using a single binary value greater than the supplied ULN of any assay to diagnose whether a patient has had an acute myocardial infarction is flawed. This is highlighted by the observed 99th centile in the study population, which is over seven times higher than the ULN recommended by the manufacturer. Furthermore, the observed frequency of hs-cTnI greater than the recommended ULN, regardless of location, in patients in whom there was no clinical suspicion of acute myocardial infarction or myocardial injury raises concerns about using a 99th centile value from a "healthy population." In particular, it might be inappropriate to apply the recommended 99th centile when managing patients who are typically older, have more comorbidities, a higher incidence of subclinical cardiac disease, and are in a worse physical condition than the healthy reference population.

The results of this study should highlight that although hs-cTnI can contribute to the diagnosis of acute myocardial infarction, frontline clinical staff should use this test in conjunction with other key factors, such as clinical history and other investigations. ${ }^{942529}$ 46-50 At present, using the 99th centile to help rule out a diagnosis of acute myocardial infarction is clear cut and is based on a "healthy" reference population. However, the recommended threshold and its application to patients presenting to hospital to rule in acute myocardial infarction is problematic, particularly when the degree of suspicion is low and other factors might contribute to the cardiac troponin concentration. Currently, the implications of detecting a hs-cTnI concentration above the supplied ULN, in terms of outcome and management, are unclear in patients in whom there is low clinical suspicion of acute myocardial infarction. A more considered approach to applying hs-cTnI concentrations would be to tailor the ULN according to the patient's baseline characteristics and comorbidities. The feasibility of using this approach, however, has not been investigated. Further data about the potential association between hs-cTnI level and cardiovascular risk are required.

\section{Limitations of this study}

There were a number of limitations. This is an observational study of a large number of consecutive patients. Therefore, the level of detail about management and diagnoses can only be obtained from the best records available for each patient, which included electronic blood request or discharge summary data, and formalised coding records. In addition, we did not examine clinical outcomes because this was not part of our objective. We also used discharge codes in our analysis for diagnosing acute myocardial infarction, but these final diagnoses were not independently verified. Finally, this study looked at hs-cTnI concentrations in 20000 patients based on a single sample for each patient; as a result, we could not differentiate between acute and chronic myocardial injury.

\section{Conclusions}

This study has shown that the 99th centile of high sensitivity troponin I concentration of the population in our hospital was substantially higher

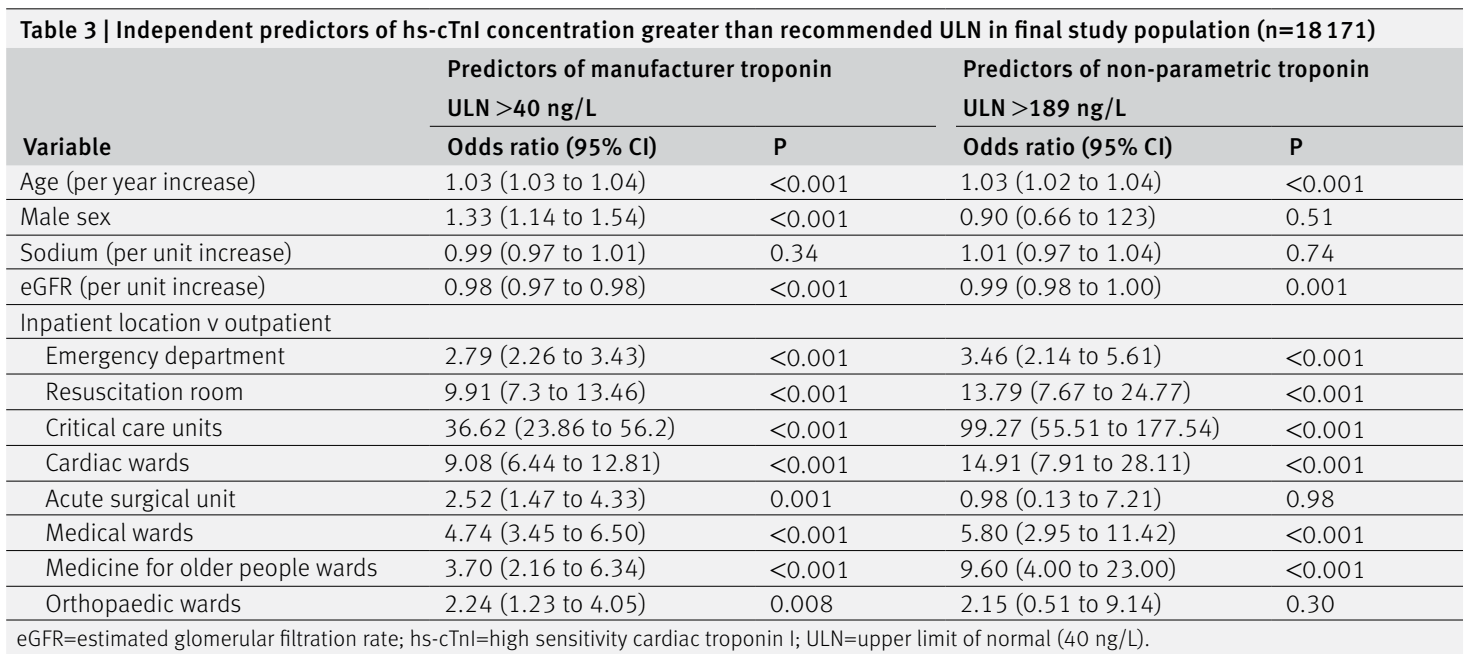


than the manufacturer's recommended ULN used in clinical practice based on the 99th centile for a healthy population. Furthermore, the 99th centile for the hospital population varied depending on the clinical setting, age and sex of the patient, and location when the test was requested; however, in all groups, a proportion of the patients had hs-cTnI concentrations greater than the recommended ULN. The study observations highlight the need for clinical staff to interpret hs-cTnI concentrations carefully and systematically when making a diagnosis of acute myocardial infarction, particularly type 1 myocardial infarction.

\section{AUTHOR AFFILIATIONS}

Coronary Research Group, University Hospital Southampton NHS Foundation Trust, Southampton, UK

${ }^{2}$ Biochemistry Department, University Hospital Southampton NHS Foundation Trust, Southampton, UK

${ }^{3}$ Emergency Medicine Department, University Hospital Southampton NHS Foundation Trust, Southampton, UK

${ }^{4}$ Coding Department, University Hospital Southampton NHS Foundation Trust, Southampton, UK

${ }^{5}$ Wessex Cardiothoracic Unit, University Hospital Southampton NHS Foundation Trust, Southampton, UK

${ }^{6}$ Keele Cardiovascular Research Group, Centre for Prognosis Research, Institute of Primary Care and Health Sciences, Keele University, Stoke on Trent, UK

${ }^{7}$ Faculty of Medicine, University of Southampton, Southampton, UK

We thank Keith Jackson, chairman of the British Cardiac Patients Association, for his assessment of the method and support for our Confidentiality Advisory Group submission.

Contributors: MM and NC performed the literature search and drew the figures. MM, RA, ZN, PC, and NC designed the study. MM, RA, SR, BO, JH, MA, ZN, AC, SC, MM, JR, IS, JW, CSK, PC, and NC collected the data. All authors contributed to data analysis, data interpretation, and writing the paper. All authors take responsibility for all aspects of the reliability and freedom from bias of the data presented and their discussed interpretation. NC, MM, PC, ZN, and RA are guarantors. The corresponding author attests that all listed authors meet authorship criteria and that no others meeting the criteria have been omitted.

Funding: Beckman Coulter (Brea, CA, USA) provided an unrestricted research grant for the study. The company had no involvement in the data collection, analysis or interpretation; trial design; or patient recruitment. The company had no role in the writing of the manuscript or decision to submit it for publication.

Competing interests: All authors have completed the ICMJE uniform disclosure form at www.icmje.org/coi_disclosure.pdf and declare: support from Beckman Coulter for the submitted work; NC received unrestricted research grants from Boston Scientific, Haemonetics, Heartflow, Beckmann Coulter; speaker fees or consultancy fees from Haemonetics, Abbot Vascular, Heartflow, and Boston Scientific; and travel sponsorship from Biosensors, Abbot, Lilly/D-S, St Jude Medical, and Medtronic.

Ethical approval: This research project was undertaken according to the principles of Good Clinical Practice and the Declaration of Helsinki. The study was approved by the local ethical committee who then referred it to the Health Research Authority UK and its independent Confidentiality Advisory Group for further approval (REC reference: 17/ SC/0042; IRAS project ID: 215262)

Data sharing: No additional data are available.

The lead author affirms that the manuscript is an honest, accurate, and transparent account of the study being reported; that no important aspects of the study have been omitted; and that any discrepancies from the study as planned (and, if relevant, registered) have been explained.

This is an Open Access article distributed in accordance with the Creative Commons Attribution Non Commercial (CC BY-NC 4.0) license, which permits others to distribute, remix, adapt, build upon this work non-commercially, and license their derivative works on different terms, provided the original work is properly cited and the use is noncommercial. See: http://creativecommons.org/licenses/by-nc/4.0/.
1 Babuin L, Jaffe AS. Troponin: the biomarker of choice for the detection of cardiac injury. CMAI 2005;173:1191-202. doi:10.1503/ cmaj/051291

2 Thygesen K, Alpert JS, Jaffe AS, et al, Joint ESC/ACCF/AHA/WHF Task Force for Universal Definition of Myocardial Infarction, Authors/ Task Force Members Chairpersons, Biomarker Subcommittee, ECG Subcommittee, Imaging Subcommittee, Classification Subcommittee, Intervention Subcommittee, Trials \& Registries Subcommittee, Trials \& Registries Subcommittee, Trials \& Registries Subcommittee, Trials \& Registries Subcommittee, ESC Committee for Practice Guidelines (CPG), Document Reviewers. Third universal definition of myocardial infarction. J Am Coll Cardiol 2012;60:1581-98. doi:10.1016/j. jacc.2012.08.001

3 Thygesen K, Alpert JS, Jaffe AS, et al, Executive Group on behalf of the Joint European Society of Cardiology (ESC)/American College of Cardiology (ACC)/American Heart Association (AHA)/World Heart Federation (WHF) Task Force for the Universal Definition of Myocardial Infarction. Fourth Universal Definition of Myocardial Infarction (2018). J Am Coll Cardiol 2018;72:2231-64. doi:10.1016/j. jacc.2018.08.1038

4 Saenger AK, Jaffe AS. Requiem for a heavyweight: the demise of creatine kinase-MB. Circulation 2008;118:2200-6. doi:10.1161/ CIRCULATIONAHA.108.773218

5 Apple FS, Collinson POIFCC Task Force on Clinical Applications of Cardiac Biomarkers. Analytical characteristics of high-sensitivity cardiac troponin assays. Clin Chem 2012;58:54-61. doi:10.1373/ clinchem.2011.165795

6 Cediel G, Gonzalez-Del-Hoyo M, Carrasquer A, Sanchez R, Boqué C, Bardají A. Outcomes with type 2 myocardial infarction compared with non-ischaemic myocardial injury. Heart 2017;103:616-22. doi:10.1136/heartjnl-2016-310243

7 Chapman AR, Adamson PD, Mills NL. Assessment and classification of patients with myocardial injury and infarction in clinical practice. Heart 2017;103:10-8. doi:10.1136/heartjnl-2016-309530

8 Melberg T, Burman R, Dickstein K. The impact of the 2007 ESC-ACCAHA-WHF Universal definition on the incidence and classification of acute myocardial infarction: a retrospective cohort study. Int I Cardiol 2010;139:228-33. doi:10.1016/j.ijcard.2008.10.021

9 Shah AS, Griffiths M, Lee KK, et al. High sensitivity cardiac troponin and the under-diagnosis of myocardial infarction in women: prospective cohort study [corrections in: BM] 2015;350:h626 and 2016;354:i4840]. BMJ 2015;350:g7873. doi:10.1136/bmj.g7873

10 Baron T, Hambraeus K, Sundström J, Erlinge D, Jernberg T, Lindahl BTOTAL-AMI study group. Impact on long-term mortality of presence of obstructive coronary artery disease and classification of myocardial infarction. Am J Med 2016;129:398-406. doi:10.1016/j. amjmed.2015.11.035

11 Shah AS, McAllister DA, Mills R, et al. Sensitive troponin assay and the classification of myocardial infarction. Am I Med 2015;128:493501.e3. doi:10.1016/j.amjmed.2014.10.056

12 Chapman AR, Shah ASV, Lee KK, et al. Long-Term Outcomes in Patients With Type 2 Myocardial Infarction and Myocardial Injury. Circulation 2018;137:1236-45. doi:10.1161/ CIRCULATIONAHA.117.031806

13 Roffi M, Patrono C, Collet JP, et al, ESC Scientific Document Group. 2015 ESC Guidelines for the management of acute coronary syndromes in patients presenting without persistent ST-segment elevation: Task Force for the Management of Acute Coronary Syndromes in Patients Presenting without Persistent ST-Segment Elevation of the European Society of Cardiology (ESC). Eur Heart J 2016;37:267-315. doi:10.1093/eurheartj/ehv320

14 Twerenbold R, Jaeger C, Rubini Gimenez M, et al. Impact of highsensitivity cardiac troponin on use of coronary angiography, cardiac stress testing, and time to discharge in suspected acute myocardial infarction. Eur Heart J 2016;37:3324-32. doi:10.1093/eurheartj/ ehw232

15 Boeddinghaus J, Nestelberger T, Twerenbold R, et al. Direct Comparison of 4 Very Early Rule-Out Strategies for Acute Myocardial Infarction Using High-Sensitivity Cardiac Troponin I. Circulation 2017:135:1597-611. doi:10.1161/ CIRCULATIONAHA.116.025661

16 Sandoval Y, Smith SW, Love SA, Sexter A, Schulz K, Apple FS. Single High-Sensitivity Cardiac Troponin I to Rule Out Acute Myocardial Infarction. Am J Med. 2017;130(9):1076-83 e1.

17 Keller T, Zeller T, Peetz D, et al. Sensitive troponin I assay in early diagnosis of acute myocardial infarction. N Engl । Med 2009;361:868-77. doi:10.1056/NEJMoa0903515

18 Keller T, Zeller T, Ojeda F, et al. Serial changes in highly sensitive troponin I assay and early diagnosis of myocardial infarction. IAMA 2011:306:2684-93. doi:10.1001/jama.2011.1896

19 Reichlin T, Hochholzer W, Bassetti S, et al. Early diagnosis of myocardial infarction with sensitive cardiac troponin assays. N Engl J Med 2009:361:858-67. doi:10.1056/NEIMoa0900428

20 Mueller M, Biener M, Vafaie M, et al. Absolute and relative kinetic changes of high-sensitivity cardiac troponin $\mathrm{T}$ in acute coronary 
syndrome and in patients with increased troponin in the absence of acute coronary syndrome. Clin Chem 2012;58:209-18. doi:10.1373/clinchem.2011.171827

21 Christ M, Bertsch T, Popp S, Bahrmann P, Heppner HJ, Muller C. Highsensitivity troponin assays in the evaluation of patients with acute chest pain in the emergency department. Clinical chemistry and laboratory medicine: CCLM / FESCC. 2011;49(12):1955-63.

22 Apple FS. A new season for cardiac troponin assays: it's time to keep a scorecard. Clin Chem 2009:55:1303-6. doi:10.1373/ clinchem.2009.128363

23 Wu AHB, Christenson RH, Greene DN, et al. Clinical Laboratory Practice Recommendations for the Use of Cardiac Troponin in Acute Coronary Syndrome: Expert Opinion from the Academy of the American Association for Clinical Chemistry and the Task Force on Clinical Applications of Cardiac Bio-Markers of the International Federation of Clinical Chemistry and Laboratory Medicine. Clin Chem 2018;64:645-55. doi:10.1373/clinchem.2017.277186

24 Eggers KM, Lind L, Venge P, Lindahl B. Factors influencing the 99th percentile of cardiac troponin I evaluated in community-dwelling individuals at 70 and 75 years of age. Clin Chem 2013;59:1068-73. doi:10.1373/clinchem.2012.196634

25 Cullen L, Greenslade JH, Carlton EW, et al. Sex-specific versus overall cut points for a high sensitivity troponin I assay in predicting 1 -year outcomes in emergency patients presenting with chest pain. Heart 2016;102:120-6. doi:10.1136/heartjnl-2015-308506

26 Kumar N, Michelis MF, DeVita MV, Panagopoulos G, Rosenstock JL. Troponin I levels in asymptomatic patients on haemodialysis using a high-sensitivity assay. Nephrol Dial Transplant 2011;26:665-70. doi:10.1093/ndt/gfa442

27 Egstrup M, Schou M, Tuxen CD, et al. Prediction of outcome by highly sensitive troponin $\mathrm{T}$ in outpatients with chronic systolic left ventricular heart failure. Am J Cardiol 2012;110:552-7. doi:10.1016/j.amjcard.2012.04.033

28 Avouac J, Meune C, Chenevier-Gobeaux C, et al. Inflammation and disease activity are associated with high circulating cardiac markers in rheumatoid arthritis independently of traditional cardiovascular risk factors. J Rheumatol 2014;41:248-55. doi:10.3899/ jrheum.130713

29 Collinson PO, Heung YM, Gaze D, et al. Influence of population selection on the 99th percentile reference value for cardiac troponin assays. Clin Chem 2012;58:219-25. doi:10.1373/ clinchem.2011.171082

30 Sandoval Y, Apple FS. The global need to define normality: the 99th percentile value of cardiac troponin. Clin Chem 2014;60:455-62 doi:10.1373/clinchem.2013.211706

31 Gore MO, Seliger SL, Defilippi CR, et al. Age- and sex-dependent upper reference limits for the high-sensitivity cardiac troponin T assay. J Am Coll Cardiol 2014;63:1441-8. doi:10.1016/j. jacc.2013.12.032

32 Shah AS, Newby DE, Mills NL. High sensitivity cardiac troponin in patients with chest pain. BMJ 2013;347:f4222. doi:10.1136/bmj. f4222

33 Saunders JT, Nambi V, de Lemos JA, et al. Cardiac troponin T measured by a highly sensitive assay predicts coronary heart disease, heart failure, and mortality in the Atherosclerosis Risk in Communities Study. Circulation 2011;123:1367-76. doi:10.1161/ CIRCULATIONAHA.110.005264

34 Thorsteinsdottir I, Aspelund T, Gudmundsson E, et al. High-Sensitivity Cardiac Troponin I Is a Strong Predictor of Cardiovascular Events and Mortality in the AGES-Reykjavik Community-Based Cohor of Older Individuals. Clin Chem 2016;62:623-30. doi:10.1373/ clinchem.2015.250811

35 Iribarren C, Chandra M, Rana JS, et al. High-sensitivity cardiac troponin I and incident coronary heart disease among asymptomatic older adults. Heart 2016;102:1177-82. doi:10.1136/ heartjnl-2015-309136

36 Melki D, Lugnegård J, Alfredsson J, et al. Implications of introducing high-sensitivity cardiac troponin T into clinical practice: data from the SWEDEHEART Registry. I Am Coll Cardiol 2015;65:1655-64. doi:10.1016/i.jacc.2015.02.044

37 Ford I, Shah AS, Zhang R, et al. High-sensitivity cardiac troponin, statin therapy, and risk of coronary heart disease. J Am Coll Cardiol 2016;68:2719-28. doi:10.1016/j.jacc.2016.10.020

38 Whelton SP, McEvoy JW, Lazo M, Coresh J, Ballantyne CM, Selvin E. High-sensitivity cardiac troponin T (hs-cTnT) as a predictor of incident diabetes in the Atherosclerosis Risk in Communities Study. Diabetes Care 2017:40:261-9 doi:10.2337/dc16-1541

39 Cavender MA, White WB, Jarolim P, et al. Serial measurement of high-sensitivity troponin I and cardiovascular outcomes in patients with type 2 diabetes mellitus in the EXAMINE Trial (examination of cardiovascular outcomes with alogliptin versus standard of care). Circulation 2017;135:1911-21. doi:10.1161/ CIRCULATIONAHA.116.024632

40 Wrigley P, Khoury J, Eckerle B, et al. Prevalence of positive troponin and echocardiogram findings and association with mortality in acute ischemic stroke. Stroke 2017;48:1226-32. doi:10.1161/ STROKEAHA.116.014561

41 Neukamm A, Einvik G, Didrik Høiseth A, et al. The prognostic value of measurement of high-sensitive cardiac troponin T for mortality in a cohort of stable chronic obstructive pulmonary disease patients. BMC Pulm Med 2016;16:164. doi:10.1186/s12890-016-0319-9

42 Willeit P, Welsh P, Evans JDW, et al. High-sensitivity cardiac troponin concentration and risk of first-ever cardiovascular outcomes in 154,052 participants. J Am Coll Cardiol 2017;70:558-68. doi:10.1016/j.jacc.2017.05.062

43 Everett BM, Zeller T, Glynn RJ, Ridker PM, Blankenberg S. Highsensitivity cardiac troponin I and B-type natriuretic Peptide as predictors of vascular events in primary prevention: impact of statin therapy. Circulation 2015;131:1851-60. doi:10.1161/ CIRCULATIONAHA.114.014522

44 Than MP, Aldous SJ, Troughton RW, et al. Detectable high-sensitivity cardiac troponin within the population reference interval conveys high 5-year cardiovascular risk: an observational study. Clin Chem 2018;64:1044-53. doi:10.1373/clinchem.2017.285700

45 Mariathas M, Curzen N. Troponin assays: developing indications. Lancet 2018;391:2398-9. doi:10.1016/S0140-6736(17)32342-5

46 Apple FS, Ler R, Murakami MM. Determination of 19 cardiac troponin I and T assay 99th percentile values from a common presumably healthy population. Clin Chem 2012;58:1574-81. doi:10.1373/ clinchem.2012.192716

47 Hammarsten O, Fu ML, Sigurjonsdottir R, et al. Troponin T percentiles from a random population sample, emergency room patients and patients with myocardial infarction. Clin Chem 2012;58:628-37. doi:10.1373/clinchem.2011.171496

48 Aw TC, Phua SK, Tan SP. Measurement of cardiac troponin I in serum with a new high-sensitivity assay in a large multi-ethnic Asian cohort and the impact of gender. Clin Chim Acta 2013;422:26-8. doi:10.1016/j.cca.2013.03.034

49 Rubini Gimenez M, Reiter M, Twerenbold R, et al. Sex-specific chest pain characteristics in the early diagnosis of acute myocardial infarction. JAMA Intern Med 2014;174:241-9. doi:10.1001/ jamainternmed.2013.12199

50 Koerbin G, Abhayaratna WP, Potter JM, et al. Effect of population selection on 99th percentile values for a high sensitivity cardiac troponin I and T assays. Clin Biochem 2013;46:1636-43. doi:10.1016/j.clinbiochem.2013.08.004

Web appendix: Supplementary material 